\title{
An analytical treatment of in-plane magnetotransport in the Falicov-Sievert model
}

\author{
Andrzej Nowojewski \\ Department of Physics, Harvard University, Cambridge, Massachusetts 02138, USA \\ Stephen J. Blundel $*$ \\ Clarendon Laboratory, Department of Physics, University of Oxford, \\ Parks Road, Oxford OX1 3PU, United Kingdom
}

(Dated: May 25, 2022)

\begin{abstract}
We derive an analytical expression which allows efficient computation of the effect of all the Fermi surface trajectories induced by a combination of Bragg scattering and magnetic breakdown on the in-plane components of the resistivity tensor. The particular network of coupled orbits which we consider was first formulated by Falicov and Sievert, who studied the problem numerically. Our approach, based upon a method used previously to derive an analytical solution for interlayer transport, allows us to show that the conductivity tensor can be written as a sum of a matrix representing the effect of total magnetic breakdown and one representing a combination of complex electronic trajectories, and we find a compact expression for the in-plane components of the resistivity tensor that can be evaluated straightforwardly.
\end{abstract}

\section{INTRODUCTION}

Magnetoresistance has long been used as a tool to study the Fermi surface of metals 1,2 . The observation of quantum oscillations provides a measure of the crosssectional area of closed pockets (due to the quantization of the Landau levels). The background magnetoresistance also contains information about all the electronic orbits induced by the magnetic field. For example, a transverse magnetoresistance saturating in high field can be evidence for the presence of closed orbits, while a magnetoresistance that increases quadratically with field can indicate open orbits. A number of effects can complicate this picture and one such is magnetic breakdown, a concept first introduced to explain a giant orbit observed in magnesium ${ }^{3}$. Because of the periodic potential in a crystal, small gaps in the dispersion sometimes open up at the Brillouin zone edge, splitting the Fermi surface into distinct sections. In low magnetic fields, electrons traverse the Fermi surface under the action of the magnetic field and Bragg scatter at the Brillouin zone edge. In a sufficiently strong magnetic field it is possible for an electron to tunnel from one segment of the Fermi surface to another ${ }^{3.4}$. This is because in high magnetic field, mixing of states between different pieces of the Fermi surface can increase the likelihood of magnetic breakdown, so that the resulting orbit more closely resembles that which would be obtained in the absence of the periodic potential $1^{5}$.

The presence of magnetic breakdown presents a challenge for the simulations of magnetoresistance. This is because at the points on the Brillouin zone edge intersected by the Fermi surface (points which we call magnetic breakdown junctions) there is a probability $p$ for magnetic breakdown to occur and this is given by $p=\exp \left(-B_{0} / B\right)$, where $B_{0}$ is the characteristic breakdown field; the problem is that under usual experimental conditions $p$ is between 0 and 1 . Thus there is a hierarchy of complex trajectories that must be summed to account for all possible contributions to the conductivity in which magnetic breakdown either does or does not occur at each magnetic breakdown junction. If a quasiparticle crosses $N$ magnetic breakdown junctions, one has to consider $2^{N}$ possible trajectories with their correct probabilistic weightings, and this complicates a direct computation of magnetoresistance since one has to sum over trajectories with arbitrarily long path lengths and hence arbitarily large values of $N$.

In order to calculate how magnetic breakdown can change the connectivity of the orbits ${ }^{6}$, and thus the resistivity, Falicov and Sievert in 1965 provided a model ${ }^{7}$ that generalised the Chambers path integral method ${ }^{8}$ to include magnetic breakdown at a finite number of points. This was used to evaluate the magnetoresistance in a number of model Fermi surfaces, although the calculations were performed numerically.

More recently, the problem of the effect of magnetic breakdown on the magnetoresistance in a network of coupled orbits was treated analytically $\underline{\underline{9}}$, though the focus of this work was on interlayer transport. This is because the newer calculations are motivated by recent experiments on quasi-two-dimensional organic metals $\frac{10-13}{1}$ in which magnetotransport experiments have been particularly illuminating 14 . In this paper we use the techniques developed in Ref. 9 (see also Ref. 15) to derive an analytical expression for the in-plane components of the resistivity tensor in the Falicov-Sievert model. This expression allows the underlying physics of the magnetotransport to be more clearly extracted and will allow a more convenient comparison with experimental data.

\section{INTRODUCTION TO THE MODEL}

In this paper, we consider the quasi-two-dimensional Fermi surface shown in Fig. 1(a) in which the dispersion is given by $E(k)=\frac{\hbar^{2}\left(k_{x}^{2}+k_{y}^{2}\right)}{2 m^{*}}-2 t_{\perp} \cos k_{z} d_{\perp}$, where $m^{*}$ 
(a)

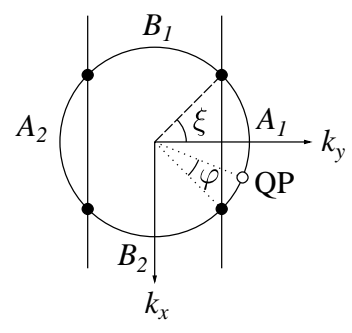

(b)

(c)

(d)

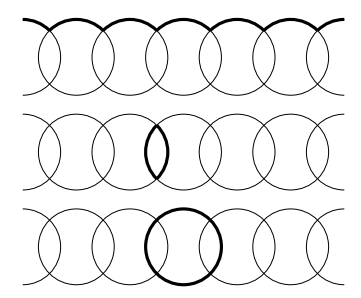

FIG. 1: (a) The Fermi surface in the $k_{x}-k_{y}$ plane showing the points where magnetic breakdown can occur which are at $\left(k_{x}, k_{y}\right)=\left( \pm k_{\mathrm{F}} \sin \xi, \pm k_{\mathrm{F}} \cos \xi\right)$ (these are called magnetic breakdown junctions). The azimuthal coordinate of a quasiparticle at the point labelled QP is $\varphi$. (b) An open orbit (comprising the repeated traversal of the $B_{1}$ section). (c) Closed orbit (the $\alpha$-orbit, comprising the repeated traversal of $A_{1}$ and $A_{2}$ ). (d) Breakdown orbit (the $\beta$-orbit, comprising $\left.A_{1}-B_{1}-A_{2}-B_{2}\right)$.

is the effective mass, $k_{\mathrm{F}}$ is the Fermi wave vector, $d_{\perp}$ is the interlayer spacing and the interlayer hopping $t_{\perp}$ is small $\left(t_{\perp} \ll \hbar k_{\mathrm{F}} / d_{\perp}\right)$. The Fermi surface consists of a cylinder with volume $\pi k_{\mathrm{F}}^{2} \times \frac{2 \pi}{d_{\perp}}$ and thus the number density of electrons is $n=k_{\mathrm{F}}^{2} / 2 \pi d_{\perp}$. The Brillouin zone edges are placed at $k_{y}= \pm k_{\mathrm{F}} \cos \xi$. Quasiparticles orbit around the Fermi surface with constant $k_{z}$ when $B$ lies along the interlayer direction. In very low $B$, because of Bragg reflection, only open orbits [Fig. 1(b)] and small closed orbits [the $\alpha$ orbit, Fig. 1(c)] occur around the distinct sections of the Fermi surface. In high $B$, mixing between the states on the two Fermi surface sections leads to magnetic breakdown at the four magnetic breakdown junctions. At these junctions a quasiparticle "tunnels" in $k$-space between the Fermi surface sections, resulting in a single large closed orbit [the $\beta$ orbit, Fig. 1(d)]. This model is equivalent to the situation outlined in Fig. 1 of Ref. 7 but the notation has been chosen to link with the physical situation relevant to a particular family of organic metals ${ }^{9}, 10$.

\section{MAGNETORESISTANCE WITH NO BRAGG SCATTERING}

In this section we review the standard calculation of magnetoresistance in the case of full magnetic breakdown (i.e. $p=1$ ) which is equivalent to assuming a very high magnetic field and ignoring any Bragg scattering. In this case we consider only the effect of a single closed breakdown orbit [i.e. the case of Fig. 1(d)]. The Boltzmann equation gives the conductivity tensor

$$
\sigma_{\alpha \beta}=e^{2} \tau g\left(E_{\mathrm{F}}\right)\left\langle v_{\alpha} \bar{v}_{\beta}\right\rangle_{\mathrm{FS}} .
$$

as an average of velocity correlations over the Fermi surface, where $\overline{v_{\beta}}$ is given by

$$
\overline{v_{\beta}}=\int_{0}^{\infty} \frac{\mathrm{e}^{-t / \tau}}{\tau} v_{\beta}[\mathbf{k}(t)] \mathrm{d} t,
$$

and $g(E)=m^{*} / d_{\perp} \pi \hbar^{2}$ is the density of states. The quasiparticle orbits lie in a plane perpendicular to the magnetic field $\mathbf{B}$ and orbit the Fermi surface with angular frequency $\omega_{\mathrm{c}}$ given by $\omega_{\mathrm{c}}=e B / \mathrm{m}^{*}$ if $\mathbf{B}$ is perpendicular to the quasi-two-dimensional planes. Writing $\varphi=\omega_{\mathrm{c}} t+$ $\varphi_{0}$, we have that

$$
\overline{v_{\beta}}=\frac{1}{\omega_{\mathrm{c}} \tau} \int_{\varphi_{0}}^{\infty} v_{\beta}(k) \mathrm{e}^{-\left(\varphi-\varphi_{0}\right) / \omega_{\mathrm{c}} \tau} \mathrm{d} \varphi
$$

and so the conductivity tensor can be written as

$$
\sigma_{\alpha \beta}=\frac{\sigma_{0}}{v_{\mathrm{F}}^{2} \pi \gamma} \int_{0}^{2 \pi} \mathrm{d} \varphi_{0} v_{\alpha} \int_{\varphi_{0}}^{\infty} v_{\beta}(k) \mathrm{e}^{-\left(\varphi-\varphi_{0}\right) / \gamma} \mathrm{d} \varphi
$$

where

$$
\gamma=\omega_{\mathrm{c}} \tau=\frac{e B \tau}{m^{*}}
$$

and

$$
\sigma_{0}=\frac{e^{2} m^{*} v_{\mathrm{F}}^{2} \tau}{2 \pi \hbar^{2} d_{\perp}}=\frac{n e \gamma}{B} .
$$

Neglecting the small effect of the interplane warping $t_{\perp}$ on orbit size, and considering only the in-plane components of the conductivity tensor $\boldsymbol{\sigma}$, we proceed as follows. Setting $v_{x}=v_{\mathrm{F}} \cos \varphi$ and $v_{y}=v_{\mathrm{F}} \sin \varphi$, and assuming full magnetic breakdown (so all the orbits just go round the cylinder with no Bragg scattering), the integral can be evaluated to give

$$
\boldsymbol{\sigma}=\frac{\sigma_{0}}{1+\gamma^{2}}\left(\begin{array}{cc}
1 & \gamma \\
-\gamma & 1
\end{array}\right)
$$

and inverting this tensor yields the resistivity tensor

$$
\boldsymbol{\rho}=\boldsymbol{\sigma}^{-1}=\sigma_{0}^{-1}\left(\begin{array}{cc}
1 & -\gamma \\
\gamma & 1
\end{array}\right),
$$

or equivalently

$$
\boldsymbol{\rho}=\left(\begin{array}{cc}
\sigma_{0}^{-1} & -B / n e \\
B / n e & \sigma_{0}^{-1}
\end{array}\right)
$$

This result is exactly what one expects from the simple theory of metals: no magnetoresistance (because $\rho_{x x}=\rho_{y y}=\sigma_{0}^{-1}$ is not field-dependent) and a simple Hall effect $\rho_{x y}=-\rho_{y x}=R_{\mathrm{H}} B$ where $R_{\mathrm{H}}=1 /$ ne. For later reference, we will denote the simple form of the resistivity tensor with no magnetic breakdown in Eq. (8) as $\boldsymbol{\rho}^{0}$.

\section{MAGNETORESISTANCE INCLUDING MAGNETIC BREAKDOWN AND BRAGG SCATTERING}

A treatment fully including both magnetic breakdown and Bragg scattering can be performed using the method 
described in Ref. 9. Setting $\nu_{\alpha}=v_{\alpha} / v_{F}$ with $\nu_{x}=\cos \varphi$ and $\nu_{y}=\sin \varphi$, Eq. (4) becomes

$$
\sigma_{\alpha \beta}=\frac{\sigma_{0}}{\pi \gamma} \int_{0}^{2 \pi} \mathrm{d} \varphi_{0} \nu_{\alpha} \mathrm{e}^{\varphi_{0} / \gamma} \int_{\varphi_{0}}^{\infty} \nu_{\beta}(k) \mathrm{e}^{-\varphi / \gamma} \mathrm{d} \varphi
$$

and with the definitions of the functions $\mathrm{E}_{\alpha}^{ \pm}(x)$

$$
\mathrm{E}_{\alpha}^{ \pm}(x)=\nu_{\alpha}(x) \mathrm{e}^{ \pm x / \gamma}
$$

the conductivity can be written

$$
\sigma_{\alpha \beta}=\frac{\sigma_{0}}{\pi \gamma} \int_{0}^{2 \pi} \mathrm{d} \varphi_{0} \mathrm{E}_{\alpha}^{+}\left(\varphi_{0}\right) \int_{\varphi_{0}}^{\infty} \mathrm{E}_{\beta}^{-}(\varphi) \mathrm{d} \varphi
$$

What makes Eq. (12) challenging to evaluate is that the integrand changes depending on the path taken by the quasiparticle which, at each magnetic breakdown junction of the orbit, can either undergo magnetic breakdown tunneling (with probability $p \equiv \mathrm{e}^{-B_{0} / B}$ ) or Bragg reflection (with probability $q=1-p)^{1,5}$. The strategy for solving this problem follows the earlier approach ${ }^{9,15}$ of separating the motion of electrons in the four different segments of the orbit and constructing recursive equations which encode all the information about the behaviour at the magnetic breakdown junctions. In this way we can write the conductivity tensor as

$$
\sigma_{\alpha \beta}=\frac{\sigma_{0}}{\pi \gamma} \boldsymbol{\lambda}_{\alpha}^{+} \cdot\left(\boldsymbol{\lambda}_{\beta}^{\text {init }}+\boldsymbol{\Gamma}(\boldsymbol{I}-\boldsymbol{\Gamma})^{-1} \cdot \boldsymbol{\lambda}_{\beta}^{-}\right)
$$

where the vectors $\boldsymbol{\lambda}_{\alpha}^{ \pm}$and $\boldsymbol{\lambda}_{\alpha}^{\text {init }}$ are given by

$$
\boldsymbol{\lambda}_{\alpha}^{ \pm}=\left(\begin{array}{c}
\int_{0}^{2 \xi} d x E_{\alpha}^{ \pm}(x) \\
a^{ \pm 1} \int_{2 \xi}^{\pi} d x E_{\alpha}^{ \pm}(x) \\
(a b)^{ \pm 1} \int_{\pi+2 \xi}^{\pi} d x E_{\alpha}^{ \pm}(x) \\
\left(a^{2} b\right)^{ \pm 1} \int_{\pi+2 \xi}^{2 \pi} d x E_{\alpha}^{ \pm}(x)
\end{array}\right)
$$

and

$$
\lambda_{\alpha}^{\text {init }}=\left(\begin{array}{c}
\int_{\varphi_{0}}^{2 \xi} d \varphi E_{\alpha}^{-}(\varphi) \\
a^{-1} \int_{\varphi_{0}}^{\pi} d \varphi E_{\alpha}^{-}(\varphi) \\
(a b)^{-1} \int_{\varphi_{0}}^{\pi+2 \xi} d \varphi E_{\alpha}^{-}(\varphi) \\
\left(a^{2} b\right)^{-1} \int_{\varphi_{0}}^{2 \pi} d \varphi E_{\alpha}^{-}(\varphi)
\end{array}\right)
$$

and the matrix $\boldsymbol{\Gamma}$ which encodes all the breakdown probabilities and scattering is given by

$$
\boldsymbol{\Gamma}=\left(\begin{array}{llll}
a & 0 & 0 & 0 \\
0 & b & 0 & 0 \\
0 & 0 & a & 0 \\
0 & 0 & 0 & b
\end{array}\right) \cdot\left(\begin{array}{llll}
0 & p & q & 0 \\
0 & q & p & 0 \\
q & 0 & 0 & p \\
p & 0 & 0 & q
\end{array}\right)
$$

where $a=\exp (-2 \xi / \gamma)$ and $b=\exp (-(\pi-2 \xi) / \gamma)$.

The integrals can be performed analytically and after much simplification (see Appendix) the conductivity can be obtained as

$$
\boldsymbol{\sigma}=\frac{\sigma_{0}}{1+\gamma^{2}}\left(\begin{array}{cc}
1 & \gamma \\
-\gamma & 1
\end{array}\right)+\Delta \frac{2 \sigma_{0}}{1+\gamma^{2}} \mathbf{X}
$$

where the parameter $\Delta$ is given by

$$
\Delta=\frac{4|\gamma|}{\pi\left(1+\gamma^{2}\right)}\left(\frac{q(1+a b) \cos ^{2} \xi}{1-b q+a\left(q+b\left(p^{2}-q^{2}\right)\right)}\right),
$$

and the matrix $\mathbf{X}$ is given by

$$
\mathbf{X}=\left(\begin{array}{cc}
\gamma^{2} \sin ^{2} \xi-\cos ^{2} \xi & -\gamma-\left(\gamma^{2}+1\right) \sin \xi \cos \xi \\
\gamma-\left(\gamma^{2}+1\right) \sin \xi \cos \xi & \gamma^{2} \cos ^{2} \xi-\sin ^{2} \xi
\end{array}\right) \text {. }
$$

Equation (17) demonstrates that the conductivity is a sum of the familiar expression assuming no Bragg scattering [i.e. Eq. (7)] and an additional term. This additional term is included by an amount controlled by $\Delta$, and it is therefore useful to plot the field dependence of this quantity. This is shown in Fig. 2(a) for the case in which $\xi=\pi / 6$ (chosen to match Fig. 1 of Ref. 7) for a range of different breakdown fields (parametrized by $\gamma_{0} \equiv \omega_{0} \tau=e B_{0} \tau / m^{*}$ ) and plotted as a function of magnetic field (parametrized by $\gamma \equiv \omega_{\mathrm{c}} \tau=e B \tau / m^{*}$ ).

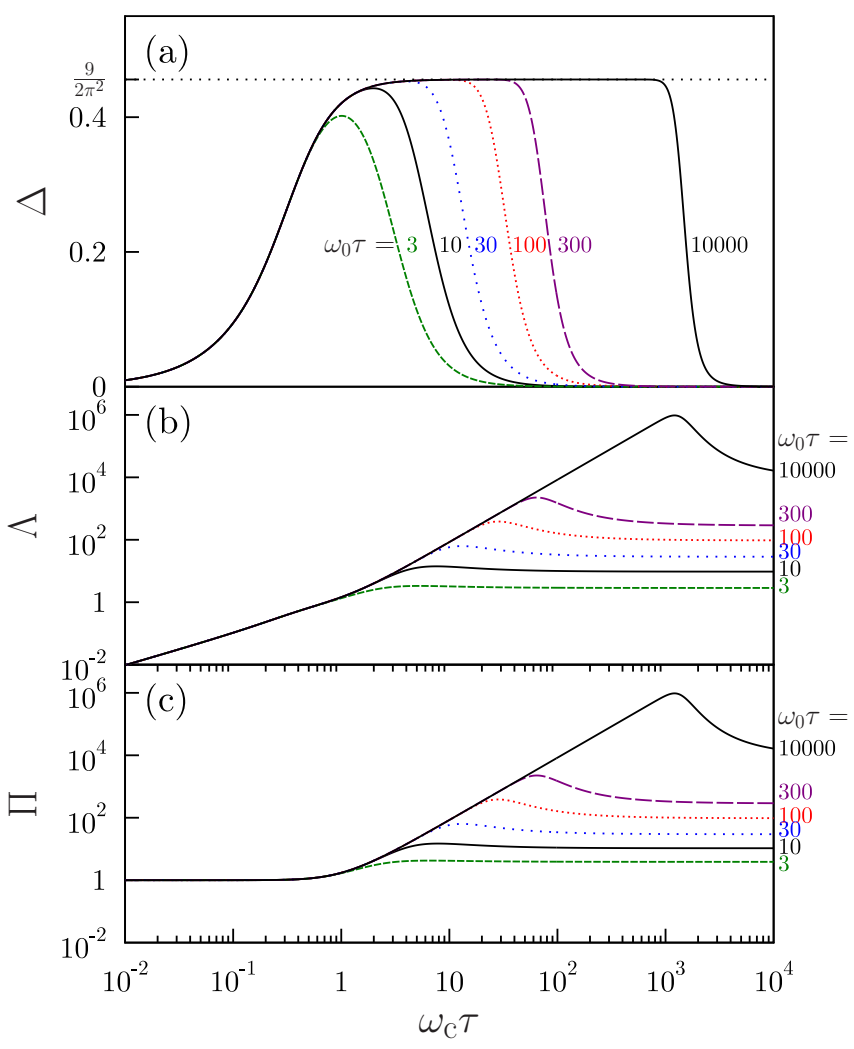

FIG. 2: (Color online.) The parameters (a) $\Delta$ [Eq. [18)], (b) $\Lambda$ [Eq. (21)] and (c) $\Pi$ [Eq. (22)].

For low field $\Delta \approx \alpha \gamma /\left(1+\gamma^{2}\right)$ where $\alpha=\left(4 \cos ^{2} \xi\right) / \pi$ and is independent of breakdown field $\gamma_{0}$. For intermediate fields, and for $\gamma_{0} \gg 1, \Delta$ rises to a plateau given by $\Delta \approx 4 \cos ^{2} \xi /[\pi(\pi-2 \xi)]=\alpha /(\pi-2 \xi)$. In our example in which $\xi=\pi / 6$, the plateau is at $\Delta \approx 9 / 2 \pi^{2}$ [see 
Fig. 2(a)]. For large fields, $\Delta$ decreases again and follows $\Delta \approx \alpha \gamma_{0} / \gamma^{2}$. Thus we expect that at very low and very high fields the approximation of no Bragg scattering (the single breakdown orbit) in Eq. (7) will work well. It works at very low fields because electrons travel only a very short distance around the Fermi surface before scattering and so orbit connectivity is largely irrelevant. It works at very high fields because then the magnetic breakdown probability is close to unity. At intermediate fields $\Delta$ is significant and the effect of magnetic breakdown and Bragg scattering is important in determining the magnetotransport.

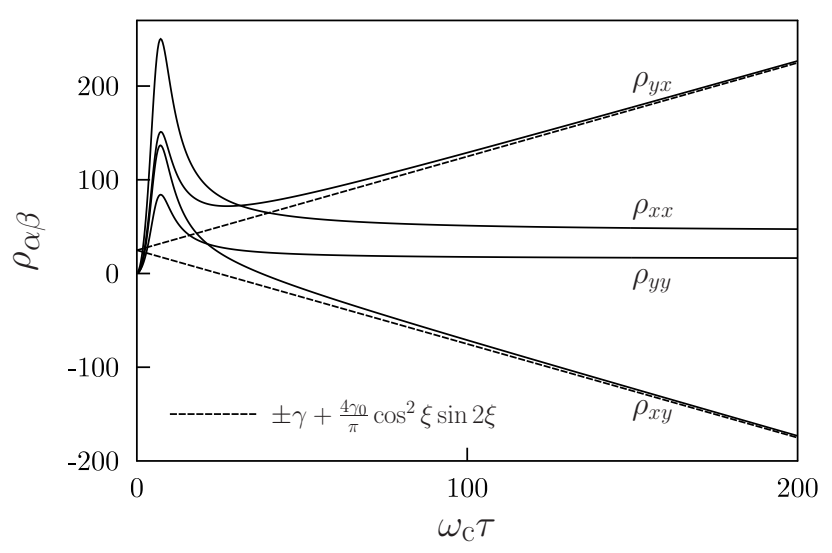

FIG. 3: The components of the resistivity tensor (plotted in units of $\sigma_{0}^{-1}$ ) calculated according to Eqs. (20,22) for the case of $\xi=\pi / 6$ and $\omega_{0} \tau=30$.

We now invert the conductivity tensor in Eq. (17) to obtain an expression for the resistivity tensor, and find

$$
\boldsymbol{\rho}=\sigma_{0}^{-1}\left(\begin{array}{cc}
\Pi+\Lambda \cos 2 \xi & -\gamma+\Lambda \sin 2 \xi \\
\gamma+\Lambda \sin 2 \xi & \Pi-\Lambda \cos 2 \xi
\end{array}\right),
$$

where

$$
\Lambda=\frac{\Delta\left(\gamma^{2}+1\right)}{1-2 \Delta}
$$

and

$$
\Pi=\frac{1+\Delta\left(\gamma^{2}-1\right)}{1-2 \Delta} .
$$

Equations (20 22) are the main results of the paper. The quantities $\Lambda$ and $\Pi$ are plotted in Fig. 2(b) and (c). In low field $(\gamma \ll 1) \Lambda \approx \alpha \gamma$ and $\Pi \approx 1$. In large field $(\gamma \gg 1) \Lambda \approx \alpha \gamma_{0}$ and $\Pi \approx 1+\alpha \gamma_{0}$. These results can be used to show that in low field the resistivity tensor is

$$
\boldsymbol{\rho}=\boldsymbol{\rho}^{0}+\sigma_{0}^{-1} \gamma \alpha \mathbf{Y}
$$

while in high fields the resistivity tensor is

$$
\boldsymbol{\rho}=\boldsymbol{\rho}^{0}+\sigma_{0}^{-1} \gamma_{0} \alpha \mathbf{Y}
$$

where the matrix $\mathbf{Y}$ contains only geometric factors and is given by

$$
\mathbf{Y}=\left(\begin{array}{cc}
1+\cos 2 \xi & \sin 2 \xi \\
\sin 2 \xi & 1-\cos 2 \xi
\end{array}\right)
$$

\section{DISCUSSION}

The components of the resistivity tensor obtained using equations (20 22) are shown in Fig. 3 for the case of $\xi=\pi / 6$ and $\omega_{0} \tau=30$. At high magnetic field the diagonal components of the resistivity tensor saturate (to a value linear in $\gamma_{0}$ ), while the off-diagonal terms approach the asymptotic values proportional to $\pm \gamma+\gamma_{0} \alpha \sin 2 \xi$ [in agreement with Eq. (24)]. As the magnetic field is decreased all resistivity tensor elements go through a maximum that becomes sharper with increasing breakdown field $\gamma_{0}$. At lower fields they decrease linearly. The position of the maximum is controlled predominantly by the behavior of $\Delta$ plotted in Fig. 2 .

The components of the resistivity tensor are plotted again in Fig. 4, though this time for a range of values of breakdown fields (parametrized by $\gamma_{0}=\omega_{0} \tau$ ). [The plot of $\rho_{x x}$ is identical to that of Fig. 1(c) in Ref. 7, demonstrating the agreement of the analytical expressions in Eqs. (20 22) and the earlier numerical work.] The difference between $\rho_{x x}$ and $\rho_{y y}$ reflects the fact that for our considered geometry in Fig. $4(\xi=\pi / 6)$ the open orbits which occur due to Bragg scattering are efficient at carrying current in the $y$-direction. This difference vanishes when $\xi=\pi / 4$.

It is worthwhile to show that the off-diagonal components of the resistivity tensor do indeed obey Onsager symmetry, i.e. $\rho_{x y}(B)=\rho_{y x}(-B)$. Onsager symmetry is dependent upon perfect microscopic reversibility (with the appropriate sign change in the magnetic field) $\stackrel{16}{\infty}$, and we find that this is obeyed even with the presence of magnetic breakdown junctions, despite the apparent "randomization" of the electron trajectory which occurs at magnetic breakdown junctions. Reversal of the magnetic field changes the way in which the sections of the Fermi surface are connected at the magnetic breakdown junctions and the result of this is that the connectivity matrix $\Gamma$ transforms into its transpose. This has the effect of making $\Delta$ invariant under a sign change of the magnetic field [and is the origin of the modulus sign of the factor of $\gamma$ in the numerator of Eq. (18)]. The net consequence of this is that Onsager symmetry is preserved.

In summary, in this paper we have derived an analytical expression for the in-plane components of the resistivity tensor that includes the effect of all the orbits induced by a combination of Bragg scattering and magnetic breakdown. Eq. (17) demonstrates that approach shows that the conductivity tensor can be written as a sum of a matrix representing the effect of total magnetic breakdown and one representing a combination of complex electronic trajectories. Our main result in Eqs. 20 22) provides a compact form for the in-plane components 

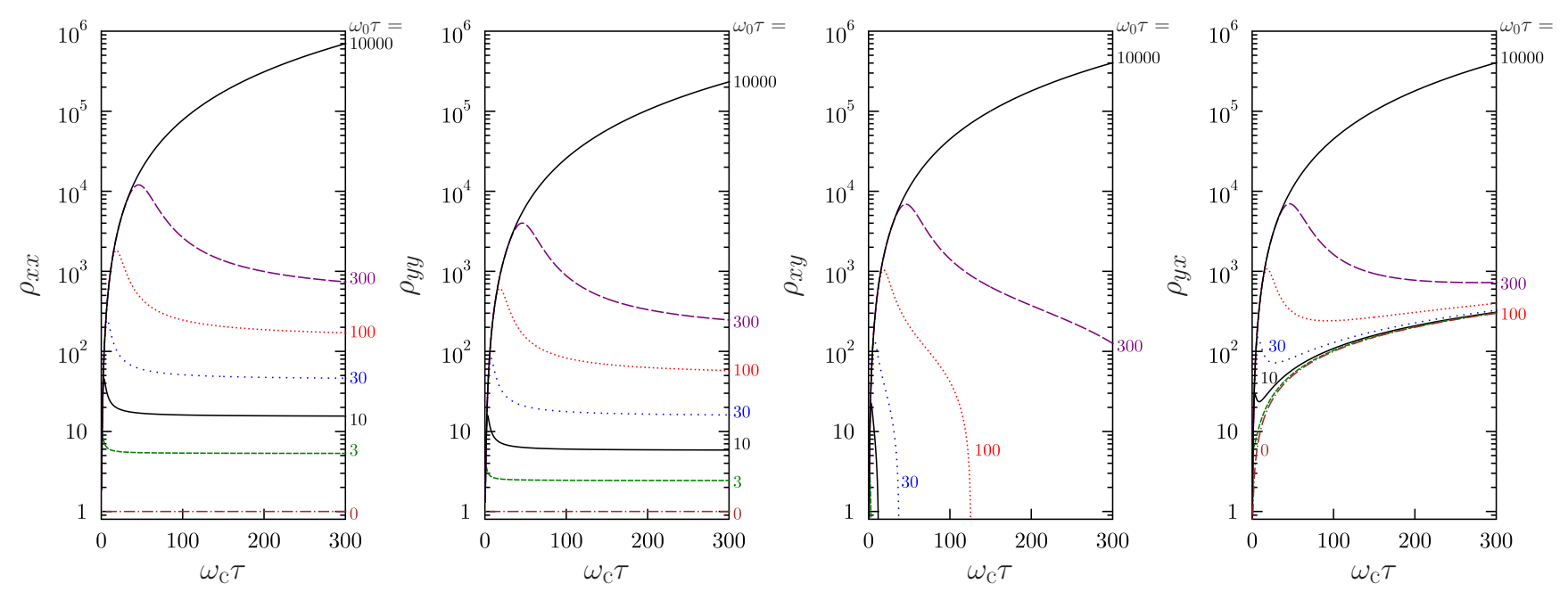

FIG. 4: (Color online.) The components of the resistivity tensor (plotted in units of $\sigma_{0}^{-1}$ ) calculated according to equations (2022) for the case of $\xi=\pi / 6$.

of the resistivity tensor for this problem which are in a convenient form to compare with experimental data.

\section{Acknowledgments}

We are grateful to EPSRC (UK) and Mansfield College, Oxford for financial support.

\section{Appendix}

The expression for conductivity from Eq. (13) is

$$
\sigma_{\alpha \beta}=\frac{\sigma_{0}}{\pi \gamma} \boldsymbol{\lambda}_{\alpha}^{+} \cdot\left(\boldsymbol{\lambda}_{\beta}^{\text {init }}+\boldsymbol{\Gamma}(\boldsymbol{I}-\boldsymbol{\Gamma})^{-1} \cdot \boldsymbol{\lambda}_{\beta}^{-}\right),
$$

and as in Ref. 9 the matrix $\boldsymbol{\Gamma}(\boldsymbol{I}-\boldsymbol{\Gamma})^{-1}$ is given by

$$
\boldsymbol{\Gamma}(\boldsymbol{I}-\boldsymbol{\Gamma})^{-1}=\frac{1}{N}\left(\begin{array}{cccc}
t & \text { apr } & \text { ars } & a^{2} p s \\
\text { abps } & w & \text { bpr } & a b p^{2} \\
a r s & a^{2} p s & t & a p r \\
b p r & a b p^{2} & \text { abps } & w
\end{array}\right)
$$

where $r=1-b q, s=q+b\left(p^{2}-q^{2}\right), N=r^{2}-a^{2} s^{2}$, $t=a^{2} s^{2}$ and $w=b\left(q r+a^{2}\left(p^{2}-q^{2}\right) s\right)$. The function
$E_{\alpha}^{ \pm}(x)$ can be simplified because $\nu_{x}=\cos \varphi, \nu_{y}=\sin \varphi$, so that

$$
\begin{aligned}
& \mathrm{E}_{x}^{ \pm}(\varphi)=\cos \varphi \mathrm{e}^{ \pm \varphi / \gamma}=\Re\left(\mathrm{e}^{(\imath \pm 1 / \gamma) \varphi}\right) \\
& \mathrm{E}_{y}^{ \pm}(\varphi)=\sin \varphi \mathrm{e}^{ \pm \varphi / \gamma}=\Im\left(\mathrm{e}^{(\imath \pm 1 / \gamma) \varphi}\right) .
\end{aligned}
$$

The vector in Eq.(14) can then be written

$$
\lambda_{\alpha}^{ \pm}=\left(\begin{array}{c}
\lambda_{\alpha \pm}^{1} \\
\lambda_{\alpha \pm}^{2} \\
\lambda_{\alpha \pm}^{3} \\
\lambda_{\alpha \pm}^{4}
\end{array}\right)=\left(\begin{array}{c}
z^{ \pm}\left(\mathrm{e}^{2 \imath \xi} a^{\mp 1}-1\right) \\
-z^{ \pm}\left(\mathrm{e}^{2 \imath \xi}+b^{\mp 1}\right) \\
-\lambda_{\alpha \pm}^{1} \\
-\lambda_{\alpha \pm}^{2}
\end{array}\right)
$$

and that in Eq.(15) as

$$
\lambda_{\alpha}^{\text {init }}=\left(\begin{array}{c}
\lambda_{\alpha i}^{1} \\
\lambda_{\alpha i}^{2} \\
\lambda_{\alpha i}^{3} \\
\lambda_{\alpha i}^{4}
\end{array}\right)=\left(\begin{array}{c}
z^{-} \mathrm{e}^{2 \imath \xi} a \\
-z^{-} b \\
-\lambda_{\alpha i}^{1} \\
-\lambda_{\alpha i}^{2}
\end{array}\right)
$$

with $z^{ \pm}=(\imath \pm 1 / \gamma)^{-1}$. The two first and two last entries of each vector differ by a minus sign because these parts of the orbit are exactly $\pi$ apart. With this identity we can readily calculate:

$$
\frac{1}{2} \lambda_{\alpha}^{+} \cdot \boldsymbol{\Gamma}(\boldsymbol{I}-\boldsymbol{\Gamma})^{-1} \cdot \lambda_{\beta}^{-}=-\left(\lambda_{\alpha+}^{1} \lambda_{\beta-}^{1}+\lambda_{\alpha+}^{2} \lambda_{\beta-}^{2}\right)+\frac{\lambda_{\alpha+}^{1} \lambda_{\beta-}^{1}(1-b q)+\lambda_{\alpha+}^{2} \lambda_{\beta-}^{2}(1+a q)+\lambda_{\alpha+}^{1} \lambda_{\beta-}^{2} a p-\lambda_{\alpha+}^{2} \lambda_{\beta-}^{1} b p}{1-b q+a\left(q+b\left(p^{2}-q^{2}\right)\right)}
$$


The initial contribution can be simplified as follows:

$$
\begin{aligned}
\lambda_{\alpha}^{+} \cdot \lambda_{\beta}^{\text {init }} & =\left(\begin{array}{c}
\lambda_{\alpha+}^{1} \\
\lambda_{\alpha+}^{2} \\
\lambda_{\alpha+}^{3} \\
\lambda_{\alpha+}^{4}
\end{array}\right) \cdot\left(\begin{array}{c}
\lambda_{\beta i}^{1} \\
\lambda_{\beta i}^{2} \\
\lambda_{\beta i}^{3} \\
\lambda_{\beta i}^{4}
\end{array}\right) \\
& =\left(\begin{array}{c}
\int_{0}^{2 \xi} d \varphi_{0} E_{\alpha}^{+}\left(\varphi_{0}\right) \\
a \int_{2 \xi}^{\pi} d \varphi_{0} E_{\alpha}^{+}\left(\varphi_{0}\right) \\
a b \int_{\pi}^{\pi+2 \xi} d \varphi_{0} E_{\alpha}^{+}\left(\varphi_{0}\right) \\
a^{2} b \int_{\pi+2 \xi}^{2 \pi} d \varphi_{0} E_{\alpha}^{+}\left(\varphi_{0}\right)
\end{array}\right) \cdot\left(\begin{array}{c}
\int_{\varphi_{0}}^{2 \xi} d \varphi E_{\beta}^{-}(\varphi) \\
a^{-1} \int_{\varphi_{0}}^{\pi} d \varphi E_{\beta}^{-}(\varphi) \\
(a b)^{-1} \int_{\varphi_{0}+2 \xi}^{\pi+2 \xi} d \varphi E_{\beta}^{-}(\varphi) \\
\left(a^{2} b\right)^{-1} \int_{\varphi_{0}}^{2 \pi} d \varphi E_{\beta}^{-}(\varphi)
\end{array}\right) \\
& =\left(\begin{array}{c}
\int_{0}^{2 \xi} d \varphi_{0} E_{\alpha}^{+}\left(\varphi_{0}\right) \\
a \int_{2 \xi}^{\pi} d \varphi_{0} E_{\alpha}^{+}\left(\varphi_{0}\right) \\
a b \int_{\pi}^{\pi+2 \xi} d \varphi_{0} E_{\alpha}^{+}\left(\varphi_{0}\right) \\
a^{2} b \int_{\pi+2 \xi}^{2 \pi} d \varphi_{0} E_{\alpha}^{+}\left(\varphi_{0}\right)
\end{array}\right) \cdot\left(\begin{array}{c}
\int^{2 \xi} d \varphi E_{\beta}^{-}(\varphi) \\
a^{-1} \int^{\pi} d \varphi E_{\beta}^{-}(\varphi) \\
(a b)^{-1} \int^{\pi+2 \xi} d \varphi E_{\beta}^{-}(\varphi) \\
\left(a^{2} b\right)^{-1} \int^{2 \pi} d \varphi E_{\beta}^{-}(\varphi)
\end{array}\right)-\left(\begin{array}{c}
\int_{0}^{2 \xi} d \varphi_{0} E_{\alpha}^{+}\left(\varphi_{0}\right) \\
\int_{2 \xi}^{\pi} d \varphi_{0} E_{\alpha}^{+}\left(\varphi_{0}\right) \\
\int_{\pi}^{\pi+2 \xi} d \varphi_{0} E_{\alpha}^{+}\left(\varphi_{0}\right) \\
\int_{\pi+2 \xi}^{2 \pi} d \varphi_{0} E_{\alpha}^{+}\left(\varphi_{0}\right)
\end{array}\right) \cdot\left(\begin{array}{c}
\int^{\varphi_{0}} d \varphi E_{\beta}^{-}(\varphi) \\
\int_{0}^{\varphi_{0}} d \varphi E_{\beta}^{-}(\varphi) \\
\int^{\varphi_{0}} d \varphi E_{\beta}^{-}(\varphi) \\
\int^{\varphi_{0}} d \varphi E_{\beta}^{-}(\varphi)
\end{array}\right) \\
& =2\left(\lambda_{\alpha+}^{1} \lambda_{\beta i}^{1}+\lambda_{\alpha+}^{2} \lambda_{\beta i}^{2}\right)-\int_{0}^{2 \pi} d \varphi_{0} E_{\alpha}^{+}\left(\varphi_{0}\right) \int^{\varphi_{0}} d \varphi E_{\beta}^{-}(\varphi)
\end{aligned}
$$

Integration of the second term then leads to

$$
\lambda_{\alpha}^{+} \cdot \lambda_{\beta}^{\mathrm{init}}=2\left(\lambda_{\alpha+}^{1} \lambda_{\beta i}^{1}+\lambda_{\alpha+}^{2} \lambda_{\beta i}^{2}\right)+\frac{\pi \gamma}{1+\gamma^{2}}\left(\begin{array}{cc}
1 & \gamma \\
-\gamma & 1
\end{array}\right)_{\alpha \beta}
$$

and hence

$$
\begin{aligned}
\sigma_{\alpha \beta} & =\frac{\sigma_{0}}{1+\gamma^{2}}\left(\begin{array}{cc}
1 & \gamma \\
-\gamma & 1
\end{array}\right)_{\alpha \beta}+\frac{2 \sigma_{0}}{\pi \gamma}\left[\left(\lambda_{\alpha+}^{1} \lambda_{\beta i}^{1}+\lambda_{\alpha+}^{2} \lambda_{\beta i}^{2}\right)-\left(\lambda_{\alpha+}^{1} \lambda_{\beta-}^{1}+\lambda_{\alpha+}^{2} \lambda_{\beta-}^{2}\right)\right] \\
& +\frac{2 \sigma_{0}}{\pi \gamma}\left[\frac{\lambda_{\alpha+}^{1} \lambda_{\beta-}^{1}(1-b q)+\lambda_{\alpha+}^{2} \lambda_{\beta-}^{2}(1+a q)+\lambda_{\alpha+}^{1} \lambda_{\beta-}^{2} a p-\lambda_{\alpha+}^{2} \lambda_{\beta-}^{1} b p}{1-b q+a\left(q+b\left(p^{2}-q^{2}\right)\right)}\right] .
\end{aligned}
$$

The final result in Eq. (17) is obtained by substituting expressions for $\lambda_{\alpha \pm}^{1}$ and $\lambda_{\alpha \pm}^{2}$ from Eq. (28) and for $\lambda_{\alpha i}^{1}$ and $\lambda_{\alpha i}^{2}$ from Eq. (29) and simplifying.

* Corresponding author: s.blundell@physics.ox.ac.uk

1 D. Shoenberg, Magnetic Oscillations in Metals, (Cambridge University Press, Cambridge, 1984).

2 A. B. Pippard, Magnetoresistance in Metals, (Cambridge University Press, Cambridge, 1984).

3 M. H. Cohen and L. M. Falicov, Phys. Rev. Lett. 7, 231 (1961).

4 E. I. Blount, Phys. Rev. 126, 1636 (1962).

5 A. B. Pippard, Proc. Roy. Soc. London A 270, 1 (1962).

${ }^{6}$ L. M. Falicov and P. R. Sievert, Phys. Rev. Lett. 12, 550 (1964).

7 L. M. Falicov and P. R. Sievert, Phys. Rev. 138, A88 (1965).

8 R. G. Chambers, Proc. Phys. Soc. A 65, 458 (1952).

9 A. Nowojewski, P. A. Goddard and S. J. Blundell, Phys. Rev. B 77, 012402 (2008).
10 P. A. Goddard, S. J. Blundell, J. Singleton, R. D. McDonald, A. Ardavan, A. Narduzzo, J. A. Schlueter, A. M. Kini and T. Sasaki, Phys. Rev. B 69, 174509 (2004).

11 W. Kang, T. Osada, Y. J. Jo, and H. Kang, Phys. Rev. Lett. 99, 017002 (2007).

12 A. F. Bangura, P. A. Goddard, J. Singleton, S. W. Tozer, A. I. Coldea, A. Ardavan, R. D. McDonald, S. J. Blundell and J. A. Schlueter, Phys. Rev. B 76, 052510 (2007).

13 W. Kang, Y. J. Jo, D. Y. Noh, K. I. Son and O.-H. Chung, Phys. Rev. B 80, 155102 (2009).

14 M. V. Kartsovnik, Chem. Rev. 104, 5737 (2004).

15 S. J. Blundell, A. Nowojewski and P. A. Goddard, Physica B 405, S134, (2010).

16 L. Onsager, Phys. Rev. 37, 405 (1931); L. Onsager, Phys. Rev. 38, 2265 (1931). 